\title{
APLICABILIDADE DA ELETROESTIMULAÇÃO COMO INIBIDOR DA ALGIA NEUROPÁTICA NA LESÃO MEDULAR
}

ELECTROESTIMULATION APPLICABILITY NEUROPATHIC PAIN INHIBITOR IN SPINAL CORD INJURY

\section{Jéssica Pereira Fialho (FIALHO, J.P.)}

Discente do Curso de Fisioterapia da Faculdade Evangélica de Ceres - GO jessfialho21@gmail.com

\section{Sara Yohana Fagundes Alves (ALVES, S.Y.F.)}

Discente do Curso de Fisioterapia da Faculdade Evangélica de Ceres - GO sarafagundesalves@hotmail.com

Taynara Gonçalves Barbosa (BARBOSA, T.G.)

Discente do Curso de Fisioterapia da Faculdade Evangélica de Ceres - GO taynaragoncalves82@gmail.com

\section{Renata Sousa Nunes (NUNES, R.S.)}

Docente do Curso de Fisioterapia da Faculdade Evangélica de Ceres - GO renatafisio8@hotmail.com

Endereço para correspondência: Av. Brasil, S/N, Qd. 13, Setor Morada Verde, 21 Ceres GO, Brasil. CEP: 76300-000 Fone: (62) 3307-7500

\section{RESUMO}

Introdução: Sabe-se que o traumatismo raquimedular é uma lesão que compromete de forma provisória ou permanente a função normal da medula espinhal e está comumente associada ao surgimento da dor neuropática. Lesões que atingem o sistema somatossensorial além de ocasionar perda da função motora podem aumentar o nível de sensibilidade álgica e um dos métodos utilizados para alívio da dor é a estimulação elétrica nervosa transcutânea. O referente método é administrado por uma máquina portátil e sua verificação é de extrema importância 
para gerar um esclarecimento abrangente tanto para indivíduos com lesão medular quanto para a comunidade científica, afim de mostrar se o método é seguro e eficaz. Sendo uma algia de difícil manuseio e diagnóstico, a dor neuropática está relacionada com a insatisfação de pacientes a tratamentos farmacológicos, não farmacológicos e cirúrgicos, necessitando assim, de uma abordagem multiprofissional incluindo Fisioterapia e Psicoterapia. Objetivo: Avaliar a efetividade da eletroestimulação como recurso terapêutico para redução da algia neuropática em indivíduos com lesão medular. Metodologia: Revisão sistemática, randomizada e descritiva, com critérios na pesquisa, inclusão e exclusão dos artigos, utilizando as bases de dados: PubMed, PubMed Central, Clinical Trials, Cochrane, LILACS, Registro Brasileiro de Ensaios Clínicos, PEDro, Scielo e BVS. Resultados e Discussão: Dez artigos foram incluídos, sendo que sete apresentam baixo risco de viés. Os resultados do estudo enfatizam os desfechos de outras duas revisões já realizadas com o intuito de avaliar a efetividade da eletroestimulação sobre a algia neuropática e/ou outras condições de dor. Conclusão: Diante da análise dos estudos encontrados não foi possível afirmar a eficácia da intervenção sobre o controle da algia neuropática em indivíduos com lesão medular.

Palavras chaves: Dor neuropática; Eletroestimulação; Lesão medular; Tratamento.

\section{ABSTRACT}

Introduction: It is known that spinal cord trauma is a lesion that temporarily or permanently compromises the normal function of the spinal cord and is commonly associated with the appearance of neuropathic pain. Lesions that affect the somatosensory system in addition to causing loss of motor function can increase the level of pain sensitivity and one of the methods used for pain relief is transcutaneous electrical nerve stimulation. The referred method is administered by a portable machine and its verification is extremely important to generate a comprehensive explanation both for individuals with spinal cord injury and for the scientific community, in order to show if the method is safe and effective. Being an algia of difficult handling and diagnosis, neuropathic pain is related to the dissatisfaction of patients with pharmacological, non-pharmacological and surgical treatments, thus requiring a multidisciplinary approach including Physiotherapy and Psychotherapy. Objective: To evaluate the effectiveness of electrostimulation as a therapeutic resource for reducing neuropathic pain in individuals with spinal cord injury. Methodology: Systematic, randomized and descriptive review, with criteria in the research, inclusion and exclusion of articles, using the databases: PubMed, PubMed Central, Clinical Trials, Cochrane, LILACS, Brazilian Registry of Clinical 
Trials, PEDro, Scielo and VHL. Results and Discussion: Ten articles were included, seven of which have a low risk of bias. The results of the study emphasize the outcomes of two other reviews already carried out in order to assess the effectiveness of electrostimulation on neuropathic pain and / or other pain conditions. Conclusion: In view of the analysis of the studies found, it was not possible to affirm the effectiveness of the intervention on the control of neuropathic pain in individuals with spinal cord injury.

Keywords: Neuropathic pain; Electro-stimulation; Spinal cord injury; Treatment.

\section{INTRODUÇÃO}

A primeira descrição sobre lesão medular (LM) vem de Edwin Smith, no século XVII a.C, a qual foi considerada uma doença que não podia ser tratada e até o momento, ainda não há métodos necessários para regeneração do tecido nervoso (JENDELOVA, 2018).

A lesão medular (LM) pode apresentar um impacto forte e tipicamente, em longo prazo, nos domínios funcionais, psicológicos e físicos de uma pessoa, sendo causado em geral por quedas, acidente de carro, armas de fogo e mergulhos. No Brasil não existem estatísticas oficiais de traumatismos na medula espinhal, pois não é obrigatório relatar tais contusões (KENNEDY; HASSON, 2017; PIZETTA et al., 2020; SILVA; JESUS, 2015).

Traumas que acometem o sistema nervoso somatossensorial podem não apenas ocasionar a perda da função, mas também aumentar a sensibilidade à dor. A dor neuropática (DN) geralmente persiste constantemente ou se manifesta com dolorosos episódios recorrentes. A causa pode ser uma doença metabólica, um tumor, infecção, uma condição neurodegenerativa, vascular ou autoimune (BURKE et al., 2017; SCHOLZ et al., 2019).

A DN pode ser provocada por estímulos sensoriais (alodinia mecânica ou dinâmica) onde a algia é pelo toque leve na pele ou pode ser espontânea (paroxística ou contínua) em suas características temporais. Tal tipo de dor é presente no e/ou abaixo do nível neurológico da lesão, mas também pode apresentar em regiões do corpo que não são afetadas pela contusão medular (DERRY et al., 2019; FRANZ et al., 2019).

A partir disso, um dos métodos não farmacológicos usado para o alívio da dor é a estimulação elétrica nervosa transcutânea (TENS), uma técnica acessível e segura para o tratamento. É administrado por uma máquina portátil com bateria que gera correntes elétricas, as quais são entregues através de eletrodos conectados com a superfície da pele (JOHNSON; MULVEY, BAGNALL, 2015). 
A importância de verificar a eficácia do TENS na dor neuropática pode ter um esclarecimento abrangente tanto para indivíduos com lesão medular quanto para a comunidade científica sobre o tratamento, mostrando também se o método é seguro e relevante para a utilização na prática clínica, sendo o mesmo utilizado como um adjuvante a outros métodos ou isoladamente sem efeitos adversos ao paciente.

Com isso, o objetivo do estudo será avaliar a efetividade da utilização da eletroestimulação como um recurso de tratamento para a redução da algia neuropática em pessoas com lesão medular, analisando sua aplicabilidade em relação a dor.

\section{METODOLOGIA}

\subsection{Desenho do estudo}

O presente estudo foi realizado por meio de uma revisão randomizada e descritiva, na qual foram estabelecidos critérios na pesquisa de artigos, inclusão e exclusão dos mesmos. O protocolo para essa revisão foi registrado International Prospective Register of Systematic Reviews (PROSPERO), com identificação n CRD42020223166.

A revisão sistemática (RS) é uma forma criteriosa de resumir as evidências científicas disponíveis que são advindas de vários ensaios clínicos, estudos de diagnóstico e prognóstico, ou de algum método em particular. Para isto, a RS utiliza uma metodologia com questões rigorosamente desenhadas e métodos para avaliar e identificar criticamente as pesquisas com grande relevância, seguida pela organização e análise de dados dos estudos que serão incluídos na revisão (ROEVER, 2017).

\subsection{Estratégia de busca}

Foram realizadas buscas nas bases de dados eletrônicas: BVS (Biblioteca Virtual em Saúde), Scielo, PubMed, PubMed Central, Clinical Trials, Cochrane, LILACS, Registro Brasileiro de Ensaios Clínicos e PEDro entre os dias 10/02/2020 e 31/10/2020, utilizando os descritores spinal cord injury, spinal trauma, electrostimulation, physiotherapy, transcutaneos electrial nerve stimulation e neuropathic pain combinados pelos operadores booleanos $A N D$ e $O R$.

Uma busca manual de rastreamento e lista de referências de artigos foi utilizada para identificar outros estudos elegíveis (Quadro 1). 
Quadro 1. Estratégia de busca nas bases de dados eletrônicas

(continua)

\begin{tabular}{|c|c|}
\hline $\begin{array}{c}\text { BASES DE } \\
\text { DADOS }\end{array}$ & ESTRATÉGIA DE BUSCA \\
\hline $\begin{array}{c}\text { PubMed } \\
\text { CENTRAL }\end{array}$ & $\begin{array}{l}\text { (Neuropathic pain AND "spinal cord injury") (Neuropathic pain AND spinal trauma AND } \\
\text { "electrostimulation") (Neuropathic pain AND Tens) (Neuropathic pain AND transcutaneous } \\
\text { stimulation) (Neuropathic pain AND spinal cord injury AND "treatment") (Neuropathic pain } \\
\text { And treatment AND electrostimulation) (Physiotherapy, neuropathic pain, tension AND spinal } \\
\text { trauma) (Tens AND neuropathic pain AND physiotherapy) (spinal cord injury AND tension) }\end{array}$ \\
\hline
\end{tabular}

(Condition or illness: Spinal cord injury Other terms: Neuropathic pain) (Condition or illness: Neuropathic pain, spinal cord trauma Other terms: Electrostimulation) (Condition or illness: Spinal cord injury Other terms: Tens) (Condition or illness: Neuropathic pain, ElectrostimulaClinical Trials tion Other terms: Conventional physiotherapy) (Condition or disease: Spinal trauma, neuropathic pain Other terms: Rehabilitation, transcutaneous electrical stimulation) (Condition or disease: Traumatic spinal cord injury, neuropathic pain Other terms: Tens) (Condition or disease: Neuropathic pain Others terms: rehabilitation, spinal cord injury)

(Neuropathic pain AND "electrical nerve stimulation") (Neuropathic pain AND electrostimulation AND "spinal cord injury") ("Electrostimulation") (*spinal cord OR trauma AND LILACS "transcutaneous stimulation" AND physiotherapy) (Neuropathic pain AND spine AND Tens E "clinical trial") (neuropathic pain and neuromodulation AND "clinical trial") (physiotherapy and spinal cord injury and electrical stimulation) (physiotherapy and electrostimulation and spinal cord trauma)

(Electrostimulation of spinal trauma) (Neuropathic pain "spinal cord injury”) (Spinal trauma) PEDro ("Transcutaneous electrostimulation”) (Neuropathic pain) (Spinal cord trauma) (Neuropathic pain with Tens) (Neuropathic pain with spinal cord injury) (* injury spinal cord with electrical stimulation) (physiotherapy with neuropathic pain)

PubMed

(Neuropathic pain AND "spinal cord injury") (spinal cord injury AND "chronic pain") (Neuropathic * AND physiotherapy AND "spinal trauma") (Neuropathic pain AND injury AND physiotherapy AND "electrostimulation") (Neuropathic pain AND Tens ) (Spinal cord injury AND electrical nerve stimulation) (Spinal cord stimulation AND traumatic spinal cord injury)

Registro Brasileiro de Ensaios Clínicos

(Spinal cord injury) (Neuropathic pain AND traumatic spinal cord injury) (Neuropathic pain in spinal cord injury) (Physiotherapy in spinal cord injury) (Neuropathic pain AND physiotherapy) (Stress in chronic pain) (Neuropathic pain AND electrostimulation) ( Traumatic spinal cord injury and electrostimulation technique) (Neuropathic pain AND physiotherapy) (Spinal cord injury AND you have AND physiotherapy) (Neuropathic pain AND you have AND treatment) (Neuropathic pain AND traumatic injury AND nerve stimulation.

(Neuropathic algia AND spinal cord injury) (Neuropathic pain AND you have "spinal cord injury") (Neuropathic pain AND electrostimulation AND physiotherapy AND "spinal cord injury") (Spinal cord injury AND treatment AND "Conventional physiotherapy") (Neuro-

Cochrane pathic pain AND nerve stimulation) transcutaneous AND spinal cord injury) (Spinal cord injury AND "electrostimulation" AND physical therapy) (* chronic pain AND tension AND spinal trauma) (Neuropathic pain AND tension AND spinal cord injury OR spinal trauma) (Neuropathic pain AND tension AND physical therapy) 


\begin{tabular}{|c|c|}
\hline $\begin{array}{l}\text { BASES DE } \\
\text { DADOS }\end{array}$ & ESTRATÉGIA DE BUSCA \\
\hline Scielo & $\begin{array}{l}\text { (Neuropathic pain AND transcutaneous electrical nerve stimulation) (Spinal cord injury AND } \\
\text { "neuropathic pain") (Neuropathic pain AND electrostimulation AND "spinal cord trauma") } \\
\text { (Neuropathic pain AND treatment AND "spinal cord trauma”) (Injury AND tension) neuro- } \\
\text { pathic pain) (neuropathic pain AND "transcutaneous electrostimulation" AND physiotherapy) } \\
\text { (* neuropathic pain AND nerve electrostimulation AND rehabilitation) }\end{array}$ \\
\hline $\begin{array}{c}\text { Biblioteca } \\
\text { Virtual em } \\
\text { Saúde (BVS) }\end{array}$ & $\begin{array}{l}\text { (Spinal cord injury And transcutaneous electrical nerve stimulation) (Spinal cord injury AND } \\
\text { "neuropathic pain") (Neuropathic pain And electrostimulation AND treatment AND "Spinal } \\
\text { cord trauma") (Neuropathic pain AND treatment AND "physiotherapy") (Injury AND neuro- } \\
\text { pathic pain) ) }\end{array}$ \\
\hline
\end{tabular}

Fonte: Elaborada pelo próprio autor

\subsection{Critérios de Elegibilidade}

Para a construção da revisão foram incluídos todos os textos em variados idiomas e com restrição de data de publicação (2015-2020), ensaios clínicos, caso-controle e revisões sistemáticas que apresentam como resultado principal o alívio da dor em pacientes portadores de algia neuropática decorrente de lesão medular, independente de idade e gênero.

Artigos de caso coorte, estudo de caso, estudos observacionais, estudos experimentais e protocolos que ainda não foram concluídos ou que fujam da temática proposta foram excluídos.

A seleção dos artigos foi realizada por três revisores de forma individual, tendo como início a leitura do título e resumo, mantendo os artigos que preencheram todos os requisitos de elegibilidade e excluindo os que não se adequam, seguida pela confirmação da elegibilidade através da leitura completa do artigo. A soma do número total de artigos encontrados em todas as bases de dados foi analisada seguida pela contabilidade e exclusão das duplicatas.

\subsection{Extração e Análise de Dados}

Os dados indispensáveis (nome do primeiro autor, ano, tipo de desfecho, ferramenta de coleta, descrição das intervenções e resultados) foram extraídos de cada artigo incluído, verificados para evitar quaisquer imprecisões ou omissões sendo modificados para uma planilha eletrônica no Microsoft Office Excel. 


\subsection{Avaliação do Risco de Viés}

O risco de viés foi avaliado utilizando a escala de PEDro, caracterizada como um instrumento para avaliação de ensaios clínicos publicados nas áreas das ciências de reabilitação. A escala possui um total de 11 itens avaliativos que, com exceção do item $\mathrm{n} 1^{\circ}$, atribui ao estudo 1 ponto para cada item satisfeito totalizando um total de 10 pontos.

Os critérios de 2 a 9 da escala analisa a validade interna do estudo enquanto os critérios 10 e 11 avaliam sua característica estatística de forma que seus resultados possam ser interpretados. Para essa revisão, adotou-se as seguintes faixas de pontuação da escala PEDro: escore de 6-10: considerou-se como alta qualidade; 4-5: qualidade moderada; e 0-3: baixa qualidade (SILVA, et al., 2017).

A avaliação foi realizada de forma independente por dois revisores (J.P.F e S.Y.F.A) e nos casos em que houve divergência, os itens discrepantes foram revistos e discutidos por um terceiro avaliador (T.G.B) para a obtenção de consenso sobre a pontuação.

\section{RESULTADOS E DISCUSSÕES}

\subsection{Identificação dos artigos}

Inúmeros estudos identificados eram ensaios clínicos realizados em animais por lesão medular induzida, a qual causava algia neuropática e cujo tratamento se dava através de estimulação elétrica nervosa transcutânea. Porém, por serem estudos em animais, esses ensaios logo eram excluídos e não acrescentavam na revisão que tem por intuito analisar efetividade da intervenção em humanos.

Foram identificados 196 artigos através da estratégia de busca pelas bases de dados utilizando de descritores combinados com operadores booleanos AND e $O R$, além de 21 artigos identificados mediante uma pesquisa manual de rastreamento de lista de referências dos quais 15 eram duplicados (Figura 1).

Dos 202 artigos rastreados 192 foram excluídos após análise dos títulos e leitura dos resumos por não abordarem o desfecho principal da temática que é o alívio da algia neuropática por estimulação elétrica nervosa transcutânea em indivíduos com lesão medular.

Com a confirmação após a leitura, foram incluídos 10 estudos na síntese qualitativa e nenhum artigo em síntese quantitativa devido à grande heterogeneidade dos artigos (Figura 1). 


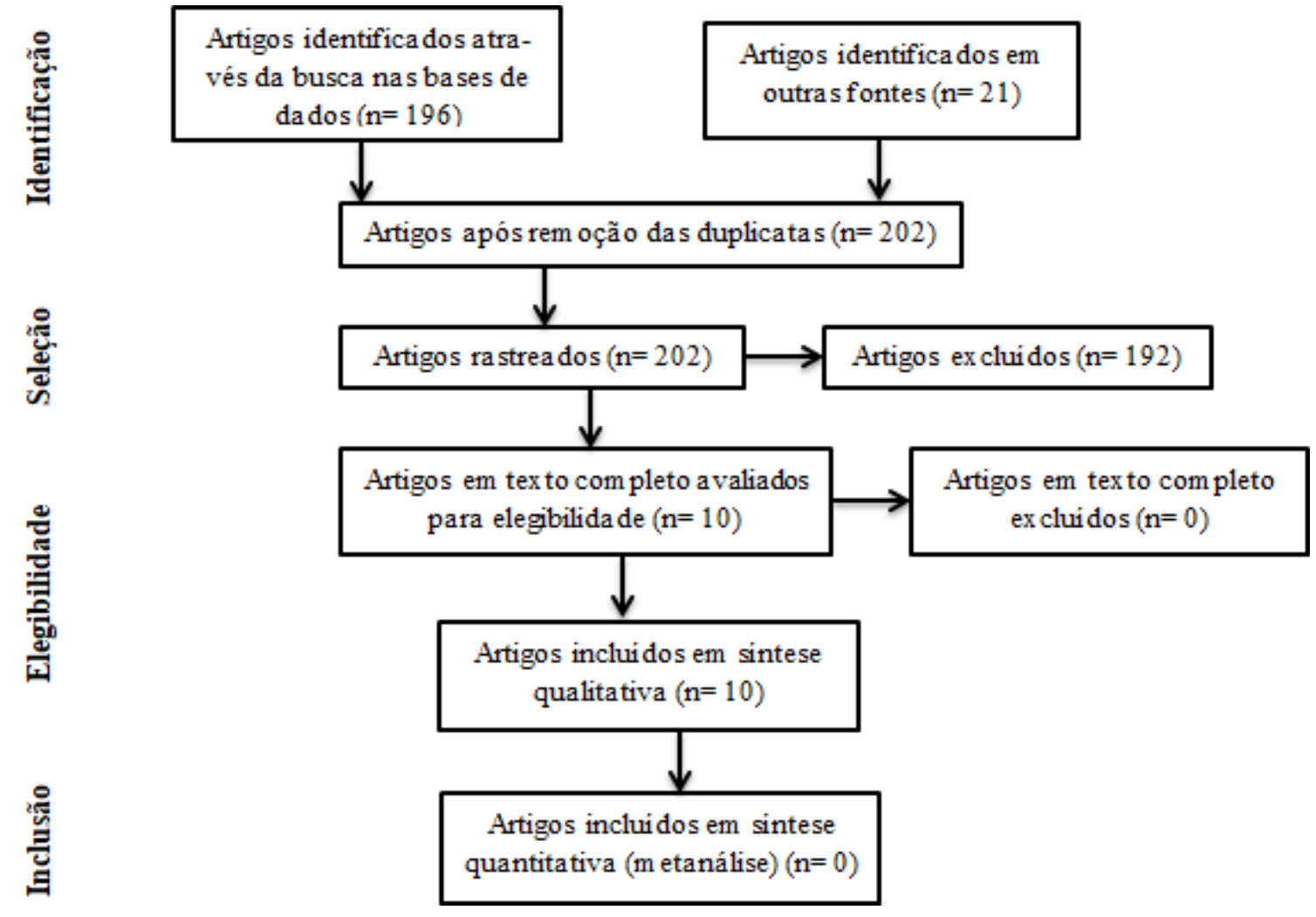

Figura 1. Fluxograma PRISMA do processo de inclusão dos artigos

Fonte: Elaborada pelo próprio autor e adaptado de Liberati et al., 2009.

\subsection{Avaliações do Risco de Viés}

$\mathrm{Na}$ avaliação do risco de viés para analisar a qualidade metodológica (Tabela 1), os artigos incluídos apresentaram média de 5,9 $\pm 2,18$ e pontuação entre 3 e 9 de 10 possíveis com 7 artigos (70\%) apresentando alta qualidade e baixo risco de viés.

O critério de acompanhamento adequado foi pontuado por todos os artigos incluídos, além desse, os critérios de distribuição cega dos sujeitos, sujeitos cegos e avaliadores cegos foram pontuados em 3 artigos (30\%), alocação aleatória, semelhança intergrupos sobre os indicadores de prognóstico e comparações intergrupos em 3 artigos (30\%) e medidas de precisão e de variabilidade em 9 artigos (90\%) (Tabela 1).

Os critérios menos pontuados foram terapeutas cegos com 2 artigos (20\%) e análise de intenção de tratar com 1 artigo (10\%) (Tabela 1). 
Tabela 1. Avaliação do risco de viés dos artigos incluídos

\begin{tabular}{lcccccccccccc}
\hline \multicolumn{1}{c}{ Autor/ Ano } & $1^{*}$ & 2 & 3 & 4 & 5 & 6 & 7 & 8 & 9 & 10 & 11 & Total \\
\hline Özkul, Çagla et al, 2015 & S & N & S & S & N & N & N & S & S & S & S & $\mathbf{6}$ \\
Vitalii, C.; Oleg, P. 2015 & S & N & S & S & S & N & S & S & S & S & S & $\mathbf{8}$ \\
Hagen, Ellen et al, 2015 & N & S & N & N & S & S & S & S & S & N & S & 7 \\
Bi, Xia et al, 2015 & S & N & S & S & N & N & S & S & S & S & S & 7 \\
C., Marleen et al, 2016 & N & N & N & N & N & N & N & S & S & N & S & $\mathbf{3}$ \\
Bilgili, Adem et al, 2016 & S & S & S & S & S & S & S & S & N & S & S & $\mathbf{9}$ \\
Li, Shengai et al, 2016 & N & S & S & S & N & N & N & S & N & S & S & $\mathbf{6}$ \\
Zeb, Amir et al, 2018 & S & N & N & N & N & N & N & S & S & N & S & $\mathbf{3}$ \\
Choi, Young-Ah et al, 2019 & N & S & S & S & S & N & N & S & S & S & N & $\mathbf{7}$ \\
H., Christine et al, 2019 & N & N & N & N & N & N & N & S & S & N & S & $\mathbf{3}$
\end{tabular}

Fonte: Elaborada pelo próprio autor.

Notas: S: Sim; N: Não. 1: Critérios de elegibilidade especificados; 2:Distribuição cega dos sujeitos; 3:Alocação aleatória; 4: Semelhança intergrupos sobre os indicadores de prognóstico; 5: Sujeitos cegos; 6: Terapeutas cegos; 7: Avaliadores cegos; 8: Acompanhamento adequado; 9: Análise de intenção de tratar; 10: Comparações intergrupos; 11: Medidas de precisão e medidas de variabilidade.

* O item 1 não contribui para a pontuação total.

\subsection{Características dos artigos}

O tamanho da amostra dos 10 artigos foi de 441 participantes variando entre 13 e 85 participantes, com idades entre 18 e 75 anos, sendo que 2 artigos não estabeleceram idade. Com relação ao gênero 8 artigos (80\%) tiveram sujeitos de ambos os sexos, 1 artigo (10\%) incluiu apenas indivíduos do sexo masculino e 1 artigo (10\%) não estabeleceu gênero dos sujeitos em seu estudo (Quadro 2).

Os principais desfechos avaliados nos artigos foram intensidade e qualidade da algia neuropática e eficácia da estimulação elétrica nervosa transcutânea. Para a mensuração da dor 10 artigos (100\%) utilizaram a Escala Visual Analógica (VAS), 1 artigo (10\%) utilizou a Escala de Dor Neuropática (NPS), 1 artigo (10\%) usou do Inventário Breve de Dor (BPI), 1 artigo (10\%) fez uso do Douleur Neuropathique en 4 Questions (DN-4), 1 artigo (10\%) utillizou da Escala de Intensidade da Dor Atual (PPI) e 3 artigos (30\%) usaram do Questionário de McGill (Quadro 2). 


\subsection{Efeitos das Intervenções}

\subsubsection{TENS e TENS versus Sham}

Dentre os estudos analisados, 2 artigos compararam somente o TENS de forma isolada e 2 artigos compararam o TENS versus TENS Sham (placebo) (Quadro 3). Há evidências que variam de alta qualidade ( 3 artigos) e baixa qualidade (1 artigo) em que a utilização do TENS possui efeitos benéficos na redução da algia neuropática em indivíduos com lesão medular (Quadro 2).

Entretanto, há evidência de alta qualidade (1 artigo) que o TENS utilizado com os estímulos é superior ao TENS Sham. Observou-se, em uma evidência de baixa qualidade que a aplicação do TENS reduz de forma significativa a dor neuropática entre sessões pré e póstratamento (Quadro 2).

\subsubsection{Comparação com outras intervenções}

Foram analisados 2 artigos comparando a utilização do TENS versus outras intervenções e encontrado evidências de alta qualidade ( 2 artigos). Desses, 1 artigo mostra a efetividade do TENS nos aspectos da qualidade, intensidade da dor e capacidade funcional do indivíduo com LM e o outro artigo verificou apenas o efeito da terapia associada em relação à algia neuropática (Quadro 2).

Em uma comparação do TENS versus Ilusão Visual em 1 artigo com evidência considerada de alta qualidade, na qual a comparação das duas técnicas pode ser usada na prática clínica como tratamento alternativo ou de suporte sendo aplicadas separadamente ou em conjunto, e um artigo, alta qualidade, a comparação do TENS versus Gabapentina demonstra eficácia da associação no tratamento da DN em lesão medular (Quadro 2).

\subsubsection{Outras Intervenções}

Em quatro artigos observou-se a utilização de terapias de estimulações convencionais como, Estimulação por Corrente Contínua Transcutânea Ativa e Simulada (tsDCS), Estimulação da Medula Espinhal (SCS), Estimulação Nervosa Periférica (PNS), Estimulação Elétrica Controlada pela Respiração (BreESTim/ESTim) e Estimulação da Medula Espinhal em Burst (Quadro 2). 
Quadro 2. Descrição dos artigos

(Continua)

\begin{tabular}{|c|c|c|c|c|c|}
\hline Autor & Participantes & Tipo de desfecho & Ferramenta de coleta & Intervenções & Resultados \\
\hline $\begin{array}{l}\text { Özkul, Çağla et al, } \\
2015\end{array}$ & $\begin{array}{l}\text { Tamanho amostral: } 24 \\
\text { Idade: não estabelecida } \\
\text { Gênero: Feminino e Masculino }\end{array}$ & $\begin{array}{l}\text { Avaliar a efetividade da } \\
\text { TENS na intensidade da } \\
\text { dor, qualidade da dor e } \\
\text { capacidade funcional em } \\
\text { pacientes com LM. }\end{array}$ & $\begin{array}{l}\text { Escala visual analógica } \\
\text { (VAS), escala de dor } \\
\text { neuropática (NPS) e o } \\
\text { inventário breve de dor } \\
\text { (BPI). }\end{array}$ & $\begin{array}{l}\text { G1 }(n=12) \text { : a ilusão foi } \\
\text { aplicada nas primeiras } 2 \\
\text { semanas, período de wash } \\
\text { out de } 1 \text { semana e, a TENS } \\
\text { foi aplicada por } 2 \text { semanas. } \\
\text { G2 }(n=12) \text { : a TENS foi } \\
\text { aplicada em primeiro lugar, } \\
1 \text { semana de wash out, em } \\
\text { seguida, \% de ilusão visual } \\
\text { (VI) foi aplicada. }\end{array}$ & $\begin{array}{l}\text { Observou-se no estudo, } \\
\text { que a intensidade da dor } \\
\text { diminuiu imediatamente } \\
\text { após as duas aplicações } \\
(V I: p=0,07 ; \text { TENS: } p= \\
0,08) \text {. Após a aplicação } \\
\text { da TENS por } 2 \text { semanas, } \\
\text { observou-se diminuição } \\
\text { significativa na maioria } \\
(p=0,02) \text { intensidade da } \\
\text { dor. }\end{array}$ \\
\hline $\begin{array}{l}\text { Vitalii, C.; Oleg, P. } \\
2015\end{array}$ & $\begin{array}{l}\text { Tamanho amostral: } 25 \\
\text { Idade: Média } 30,38 \pm 6,91 \\
\text { Gênero: Feminino e Masculino }\end{array}$ & $\begin{array}{l}\text { Investigar o efeito da } \\
\text { TENS de baixa frequência } \\
\text { no tratamento da DN em } \\
\text { pacientes com LM. }\end{array}$ & $\begin{array}{l}\text { Escala visual analógica } \\
\text { (VAS). }\end{array}$ & $\begin{array}{l}\text { Os pacientes foram desig- } \\
\text { nados aleatoriamente para } \\
\text { grupos de estudo e contro- } \\
\text { le. O grupo de estudo foi } \\
\text { tratado com } 30 \text { min de } \\
\text { TENS diariamente por } 10 \\
\text { dias, enquanto o grupo } \\
\text { controle com } 30 \text { min de } \\
\text { TENS placebo. }\end{array}$ & $\begin{array}{l}\text { Este estudo revelou que } \\
\text { no tratamento da DN de } \\
\text { pacientes com LM, a } \\
\text { TENS pode ser eficaz em } \\
\text { combinação com gaba- } \\
\text { pentina. TENS pode } \\
\text { efetivamente comple- } \\
\text { mentar o tratamento } \\
\text { farmacológico em paci- } \\
\text { entes com LME e DN. } \\
\text { Uma redução significati- } \\
\text { va dos valores de VAS } \\
\text { do que no grupo contro- } \\
\text { le. }\end{array}$ \\
\hline $\begin{array}{l}\text { Hagen, Ellen et al, } \\
2015\end{array}$ & $\begin{array}{l}\text { Tamanho amostral: } 90 \\
\text { Tempo de lesão: } 1,6 \text { e } 12 \text { meses } \\
\text { Gênero: Feminino e Masculino }\end{array}$ & $\begin{array}{l}\text { Discutir a eficácia da inter- } \\
\text { venção no estudo clínico. }\end{array}$ & $\begin{array}{l}\text { Escala visual analógica } \\
\text { (VAS) e Questionário de } \\
\text { McGill. }\end{array}$ & $\begin{array}{l}\text { Aplicado a TENS de baixa } \\
\text { frequência sobre a área } \\
\text { álgica em todos os pacien- } \\
\text { tes durante } 12 \text { meses. }\end{array}$ & $\begin{array}{l}\text { Oitenta e oito pacientes } \\
\text { completaram o acompa- } \\
\text { nhamento de } 12 \text { meses. } \\
\text { O resultado demonstrou } \\
\text { um efeito favorável na } \\
\text { dor durante sua aplica- } \\
\text { ção. }\end{array}$ \\
\hline
\end{tabular}


Quadro 2. Descrição dos artigos

(Continuação)

\begin{tabular}{|c|c|c|c|c|c|}
\hline Autor & Participantes & Tipo de desfecho & Ferramenta de coleta & Intervenções & Resultados \\
\hline Bi, Xia et al, 2015 & $\begin{array}{l}\text { Tamanho amostral: } 52 \\
\text { Idade: } 58 \text { a } 65 \text { anos } \\
\text { Gênero: Feminino e Mas- } \\
\text { culino }\end{array}$ & $\begin{array}{l}\text { Efetividade da TENS na } \\
\text { dor de pacientes com } \\
\text { LME. }\end{array}$ & $\begin{array}{l}\text { Escala visual analógica } \\
\text { (VAS) e o Questionário } \\
\text { de dor McGill. }\end{array}$ & $\begin{array}{l}\text { G1: pacientes tratados } \\
\text { com TENS, foi utilizado } \\
\text { uma máquina Physiomed } \\
\text { e } 2 \text { eletrodos foram colo- } \\
\text { cados na região álgica } \\
\text { (paramêtros: frequência } \\
\text { 2Hz; duração de pulso: } \\
\text { menos de 200ms e ampli- } \\
\text { tude de pulso: } 50 \mathrm{~mA} \text { ). } \\
\text { G2: pacientes tratados } \\
\text { com TENS simulada } \\
\text { (eletrodos alocados, mas } \\
\text { nenhum estímulo foi acio- } \\
\text { nado). } \\
\text { Protocolo: } 20 \text { min, } 3 x \text { na } \\
\text { semana por } 12 \text { semanas. }\end{array}$ & $\begin{array}{l}\text { Dez dos } 62 \text { participantes } \\
\text { foram considerados inele- } \\
\text { gíveis para o estudo, } 8 \text { não } \\
\text { preencheram os critérios } \\
\text { de inclusão e } 2 \text { tinham } \\
\text { experiência anterior com } \\
\text { TENS. No total } 48 \text { pacien- } \\
\text { tes completaram as } 12 \\
\text { semanas de tratamento. } \\
\text { Quatro pacientes ( } n=2 \\
\text { grupo TENS e } n=2 \text { grupo } \\
\text { controle) perderam o } \\
\text { acompanhamento devido } \\
\text { doença ou sua retirada. } \\
\text { Após a intervenção, houve } \\
\text { uma diferença significati- } \\
\text { va nos escores de intensi- } \\
\text { dade da dor dos grupos } \\
\text { TENS e controle } \\
\text { ( } p<0,05 \text { ). }\end{array}$ \\
\hline C., Marleen et al, 2016 & $\begin{array}{l}\text { Tamanho amostral: } 52 \\
\text { Idade: maiores de } 18 \text { anos } \\
\text { Gênero: não estabelecido }\end{array}$ & $\begin{array}{l}\text { Avaliar a Dor Neuropática } \\
\text { (DN). }\end{array}$ & $\begin{array}{l}\text { Escala visual analógica } \\
\text { (VAS), McGill Pain } \\
\text { Question-naire (MPQ) e o } \\
\text { VAS para qualidade de } \\
\text { vida (QV). }\end{array}$ & $\begin{array}{l}\text { No total } 40 \text { pacientes } \\
\text { foram recrutados para o } \\
\text { estudo. A amplitude para } \\
\text { estimulação tônica variou } \\
\text { entre } 0,4 \text { e } 19 \mathrm{~mA} \text {, com } \\
\text { largura de pulso entre } 100 \\
\text { e } 500 \text { ls e frequências } \\
\text { entre } 30 \text { e } 120 \mathrm{~Hz} \text { durante } \\
2 \text { semanas. }\end{array}$ & $\begin{array}{l}\text { A pontuação VAS média } \\
\text { para dor foi menor duran- } \\
\text { te a estimulação burst de } \\
\text { alta }(40, p=0,013) \text { e baixa } \\
\text { amplitude }(42, p=0,053) \\
\text { em comparação com a } \\
\text { estimulação tônica (52). } \\
\text { No nível individual, 58\% } \\
\text { tiveram redução da algia. }\end{array}$ \\
\hline
\end{tabular}


Quadro 2. Descrição dos artigos

(Continuação)

\begin{tabular}{|c|c|c|c|c|c|}
\hline Autor & Participantes & Tipo de desfecho & Ferramenta de coleta & Intervenções & Resultados \\
\hline Bilgili, Adem et al, 2016 & $\begin{array}{l}\text { Tamanho de amostra: } 30 \\
\text { Idade: Média } 49,07 \pm \\
\text { 10,26 } \\
\text { Gênero: Feminino e Mas- } \\
\text { culino }\end{array}$ & $\begin{array}{l}\text { Investigar o efeito da } \\
\text { TENS na recuperação } \\
\text { clínica de pacientes com } \\
\text { síndrome de dor regional } \\
\text { complexa. }\end{array}$ & $\begin{array}{l}\text { Escala visual analógica } \\
\text { (VAS), escala de avalia- } \\
\text { ção de sinais e sintomas } \\
\text { neuropáticos (LANSS) e } \\
\text { LEEDS e o Douleur Neu- } \\
\text { ropathique en } 4 \text { questions } \\
\text { (DN-4) para avaliar a dor. }\end{array}$ & $\begin{array}{l}\text { G1: TENS + banho de } \\
\text { contraste + banho de hi- } \\
\text { dromassagem + programa } \\
\text { de exercícios. } \\
\text { G2: SHAM TENS + ba- } \\
\text { nho de contraste + banho } \\
\text { de hidromassagem + pro- } \\
\text { grama de exercícios. } \\
\text { TENS convencional: } \\
\text { frequência } 100 \mathrm{~Hz} \text {, dura- } \\
\text { ção de pulso: } 50-100 \mathrm{~ms} \text {. } \\
\text { SHAM TENS: eletrodos } \\
\text { posicionados de maneira } \\
\text { semelhante sem corrente } \\
\text { fornecida. Banho de con- } \\
\text { traste: } 38^{\circ} \mathrm{C} 4 \text { minutos. } \\
\text { Banho de hidromassagem: } \\
37^{\circ} \mathrm{C} 15 \text { minutos. }\end{array}$ & $\begin{array}{l}\text { Melhorias significativas } \\
\text { foram alcançadas nos } \\
\text { escores de dor espontânea } \\
\text { e neuropática, edema, } \\
\text { ROM e funcional, capaci- } \\
\text { dade em ambos os grupos } \\
(p<0,05) \text {. No entanto, a } \\
\text { melhoria em relação à dor } \\
\text { avaliada por LANSS foi } \\
\text { maior no grupo G1. }\end{array}$ \\
\hline Li, Shengai et al, 2016 & $\begin{array}{l}\text { Tamanho da amostra: } 13 \\
\text { Idade: } 18 \text { a } 75 \text { anos } \\
\text { Gênero: Feminino e Mas- } \\
\text { culino }\end{array}$ & $\begin{array}{l}\text { Avaliar a Dor neuropática } \\
\text { e analgesia em pacientes } \\
\text { com LM. }\end{array}$ & $\begin{array}{l}\text { Escala visual analógica } \\
\text { (VAS). }\end{array}$ & $\begin{array}{l}\text { Exp (1): um total de } 120 \\
\text { estímulos elétricos duran- } \\
\text { te o BreEStim ou o EStim } \\
\text { foram entregues a todos } \\
\text { os sujeitos em uma ordem } \\
\text { aleatória com pelo menos } \\
3 \text { dias de intervalo. } \\
\text { Exp (2): um subconjunto } \\
\text { de } 7 \text { indivíduos recebeu } \\
\text { uma dose adicional e } \\
\text { maior de BreEStim (120 e } \\
240 \text { estímulos). }\end{array}$ & $\begin{array}{l}\text { No Exp 1, ambos BreES- } \\
\text { tim e EStim mostraram } \\
\text { efeitos analgésicos signi- } \\
\text { ficativos. A redução na } \\
\text { pontuação VAS foi signi- } \\
\text { ficativamente maior após } \\
\text { BreEStim }(2,6 \pm 0,3) \text { do } \\
\text { que após EStim }(1,9 \pm 1 \\
\text { hora) }(p=0,04) \text {. No Exp } \\
2, \text { BreEStim120 e BreES- } \\
\text { tim240 tiveram grau e } \\
\text { duração de analgésicos. }\end{array}$ \\
\hline
\end{tabular}


Quadro 2. Descrição dos artigos

(Continuação)

\begin{tabular}{|c|c|c|c|c|c|}
\hline Autor & Participantes & Tipo de desfecho & Ferramenta de coleta & Intervenções & Resultados \\
\hline Zeb, Amir et al, 2018 & $\begin{array}{l}\text { Tamanho amostral: } 60 \\
\text { Idade: } 20 \text { a } 60 \text { anos } \\
\text { Gênero: Feminino e Mas- } \\
\text { culino }\end{array}$ & $\begin{array}{l}\text { Determinar eficácia da } \\
\text { TENS no tratamento da } \\
\text { DN em pacientes com } \\
\text { LM. }\end{array}$ & $\begin{array}{l}\text { Escala visual analógica } \\
\text { (VAS). }\end{array}$ & $\begin{array}{l}\text { TENS com alta frequência } \\
\text { de } 80 \mathrm{~Hz} \text { aplicada em } \\
\text { pacientes com LM. Ses- } \\
\text { sões de } 45 \text { minutos, sendo } \\
\text { 2x por dia (matutino e } \\
\text { noturno), por } 4 \text { dias con- } \\
\text { secutivos (segunda, terça, } \\
\text { quarta e quinta-feira) com } \\
\text { duração de } 8 \text { semanas. }\end{array}$ & $\begin{array}{l}\text { A intensidade da dor na } \\
\text { VAS no início do estudo } \\
\text { foi de } 6,45 \pm 1,09 \text {, após a } \\
\text { intervenção no dia } 1 \text { da } \\
\text { semana } 1 \text {. A intensidade } \\
\text { média da dor diminuiu } \\
\text { para } 3,48 \pm 1,91 \text { no dia } 4 \\
\text { da semana } 1 \text {. Durante as } \\
\text { sessões consecutivas de } \\
\text { aplicação da TENS, a } \\
\text { intensidade diminuiu de } \\
\text { forma linear e houve dife- } \\
\text { rença significativa } \\
\text { ( } p<0,05 \text { ) entre sessões pré } \\
\text { e pós-tratamento. }\end{array}$ \\
\hline $\begin{array}{l}\text { Choi, Young-Ah et al, } \\
2019\end{array}$ & $\begin{array}{l}\text { Tamanho amostral: } 10 \\
\text { Idade: } 20 \text { a } 60 \text { anos } \\
\text { Feminino e Masculino }\end{array}$ & $\begin{array}{l}\text { Avaliar a DN em pessoas } \\
\text { com LM após aplicação } \\
\text { de estimulação espinhal } \\
\text { por corrente contínua } \\
\text { transcutânea (tsDCS). }\end{array}$ & $\begin{array}{l}\text { Escala visual analógica } \\
\text { (VAS) e escala de intensi- } \\
\text { dade da dor atual (PPI). }\end{array}$ & $\begin{array}{l}\text { G1: participantes subme- } \\
\text { tidos ao procedimento de } \\
\text { tsDCS ativo. } \\
\text { G2: participantes subme- } \\
\text { tidos ao procedimento de } \\
\text { tsDCS simulado. }\end{array}$ & $\begin{array}{l}\text { Os resultados não mostra- } \\
\text { ram nenhuma diferença } \\
\text { significativa pré e pós- } \\
\text { tratamento na intensidade } \\
\text { da dor entre os grupos } \\
\text { tsDCS ativo e simulado. } \\
\text { Os resultados sugerem } \\
\text { que uma única sessão de } \\
\text { tsDCS não tem efeito } \\
\text { analgésico significativo } \\
\text { em indivíduos com LME. }\end{array}$ \\
\hline
\end{tabular}


Quadro 2. Descrição dos artigos

(Continuação)

\begin{tabular}{|c|c|c|c|c|c|}
\hline Autor & Participantes & Tipo de desfecho & Ferramenta de coleta & Intervenções & Resultados \\
\hline H., Christine et al, 2019 & $\begin{array}{l}\text { Tamanho amostral: } 85 \\
\text { Idade: Média } 48,9 \pm 12,7 \\
\text { e } 45,4 \pm 8,4 \\
\text { Gênero: Masculino }\end{array}$ & $\begin{array}{l}\text { Avaliar a intensidade da } \\
\text { dor contínua, limiares de } \\
\text { dor e modulação de dor } \\
\text { condicionada. }\end{array}$ & $\begin{array}{l}\text { Escala visual analógica } \\
\text { (VAS), teste sensorial } \\
\text { quantitativo (DFNS- } \\
\text { protocolo). }\end{array}$ & $\begin{array}{l}15 \text { participantes do SCS e } \\
13 \text { do PNS foram incluí- } \\
\text { dos de forma não rando- } \\
\text { mizada. Portanto, deter- } \\
\text { minado a extremidade } \\
\text { com maior intensidade de } \\
\text { dor durante desativação } \\
\text { como área de teste. Mais } \\
\text { frequentemente } 1 \times 8 \text { deri- } \\
\text { vações ( } S C S: n=11 ; \text { PNS: } \\
n=4) \text { e } 1 \times 4 \text { derivações } \\
(S C S: n=2 ; \text { PNS: } n=6) \\
\text { foram ativadas. Faixa de } \\
\text { amplitude SCS: } 0,7 \text { a } 4,6 \\
\text { V, PNS: } 0,2 \text { a } 2,05 \mathrm{~V} ; \\
\text { alcance de frequência } \\
\text { SCS: } 60 \text { a } 120 H z, \text { PNS: } \\
60 \text { a } 130 H z ; \text { alcance de } \\
\text { duração do pulso SCS: } \\
120 \text { a } 450 \mu \text { s, PNS: } 100 \text { a } \\
450 \mu \text { s). PNS foi forneci- } \\
\text { do uma vez no nervo } \\
\text { femoral e } 4 \text { vezes em cada } \\
\text { nervo ulnar, mediano e } \\
\text { radial. }\end{array}$ & $\begin{array}{l}\text { Na condição ON, a inten- } \\
\text { sidade da dor diminuiu } \\
\text { (escala de avaliação nu- } \\
\text { mérica SCS: } 6,05 \pm 2,1 \text { vs. } \\
3,7 \pm 2,3, P<0,01 ; P N S \text { : } \\
6,2 \pm 1,4 \text { vs. } 4 \pm 1,9 \\
p<0,01 \text { ), mas isso não se } \\
\text { correlacionou com qual- } \\
\text { quer sensor de único pa- } \\
\text { râmetro. No entanto, para } \\
\text { SCS, o número total de } \\
\text { parâmetros indicando } \\
\text { hiperalgesia foi significa- } \\
\text { tivamente reduzido na } \\
\text { condição ON (45 vs. } 23 \text {, } \\
p=0,001 \text { ). Um efeito de } \\
\text { CPM menor na condição } \\
\text { OFF correlacionado com } \\
\text { uma maior melhoria de } \\
\text { COM durante a estimula- } \\
\text { ção. }\end{array}$ \\
\hline
\end{tabular}

Fonte: Elaborada pelo próprio autor.

Nota: LM: Lesão medular; LME: Lesão medular espinhal; DN: dor neuropática; TENS: estimulação elétrica nervosa transcutânea; VAS: escala visual analógica; NPS: escala de dor neuropática; BPI: inventário breve de dor; G1: grupo 1; G2: grupo 2; VI: ilusão visual; MPQ: McGill pain question-naire; QV: qualidade de vida; LANSS: escala de avaliação de sinais e sintomas; DN-4: Douleur neuropathique em 4 questions; NRS: escala de classificação numérica; PPI: intensidade da dor atual. 
Há evidências que variam de baixa (2 artigos) a alta qualidade (2 artigos) que demonstram o nível da efetividade que a estimulação convencional causa perante a dor neuropática, tendo aspectos avaliados quanto a analgesia, intensidade da dor contínua, limiares e modulação da algia condicionada e avaliação da dor neuropática em LM (Quadro 2).

A evidência considerada de alta qualidade, demonstrou que a BreESTim (1 artigo) é um tratamento não farmacológico alternativo e eficaz para algia neuropática em pacientes que apresentam LM e tsDCS (1 artigo) aponta que a terapia não indica diferença significativa de pré e pós no tratamento da dor (Quadro 2).

O estudo de baixa qualidade revela que a SCS em burst é, em geral mais eficaz que a estimulação tônica, visualizando assim uma VAS média para algia e a SCS/PNS, modulam circuitos centrais podendo apresentar efeitos benéficos no tratamento álgico (Quadro 2).

Os resultados do presente estudo reforçam desfechos com outras duas revisões (sistemática e bibliográfica) que avaliaram os efeitos do TENS sobre a dor neuropática e outras condições de dor (GIBSON; WNAD; CONNELL, 2017; MOKHTARI et al., 2020).

Uma diretriz de prática clínica para tratamento e reabilitação da algia neuropática em lesão medular de origem traumática ou não traumática ressalta que a terapia com a Estimulação Elétrica Nervosa Transcutânea (TENS) é uma recomendação de quarta linha com qualidade de evidência baixa e força de recomendação fraca. Breves revisões de Harvey, Glinsky e Bowden (2016) indicam o TENS, porém, a qualidade da evidência é moderada (GUY et al., 2016).

Em outra diretriz de Sociedade Médica Alemã para lesão medular faz recomendações quanto ao uso do TENS no tratamento da dor neuropática com um bom grau de recomendação e força de consentimento forte em casos onde outras opções de intervenções estiverem disponíveis ao paciente (FRANZ et al., 2019).

Desse mesmo modo, Kong, Gozani (2018) em seu estudo revela um resultado satisfatório, onde o TENS de alta frequência é uma opção eficaz para tratar a dor crônica em vários locais fornecendo ao paciente analgesia generalizada, decorrentes da ativação da inibição da algia. Esse processo causa uma redução clinicamente na intensidade, interferência da dor, humor e atividade. 
Este estudo aborda a aplicação da eletroestimulação na algia neuropática em lesionados medulares, o que difere de estudos voltados à condições como, a análise de efeitos da terapia sobre a dor, que é um desfecho importante dentro da condição analisada.

Deste modo, esses resultados podem ser utilizados na prática clínica para o manejo da algia neuropática em pacientes com lesão medular. Faz-se importante a presença de evidências sobre a efetividade da intervenção da Estimulação Elétrica Nervosa Transcutânea no manejo destes pacientes que contribuam para a redução da algia e para a melhora da qualidade de vida.

A confiança dentro desta estimativa de efeito se torna um tanto quanto limitada, tendo em vista as dificuldades que os pesquisadores encontram ao realizar a pesquisa por evidências publicadas sobre a efetividade da técnica devido grande parte dos estudos serem realizados em animais.

\section{CONCLUSÃO}

Relatou-se nesta revisão sobre qual seria a eficácia do TENS na inibição da algia neuropática. Porém, a qualidade de evidência é muito baixa, ou seja, não se é capaz de afirmar a efetividade da intervenção no controle da dor em pacientes com LM.

Diante do que foi analisado nos artigos encontrados para o estudo, recomenda-se a realização de ensaios clínicos em humanos, estudos de alta qualidade metodológica que avaliem melhor os efeitos a curto e longo prazo da terapia TENS na dor neuropática em lesão medular. Os projetos de estudos futuros podem contribuir para redução das incertezas quanto a eficácia desta modalidade de tratamento.

\section{REFERÊNCIAS}

BI, X. et al. Effects of transcutaneous electrical nerve stimulation on pain in patients with spinal cord injury: a randomized controlled trial. Journal of Physical Therapy Science, v. 27, p. 23-25, 2015. Disponível em: < https://pubmed.ncbi.nlm.nih.gov/25642029/>. Acesso em: 24 de Outubro de 2020.

BILGILI, A. et al. The effectiveness of transcutaneous electrical nerve stimulation in the management of patients with complex regional pain syndrome: A randomized, doubleblinded, placebo-controlled prospective study. Journal of Back and Musculoskeletal Rehabilitation, v. 29, p. 661-671, 2016. Disponível em: <https://pubmed.ncbi.nlm.nih. gov/26922847/>. Acesso em: 21 de Setembro de 2020. 
BURKE, D. et al. Prevalência de dor neuropática após lesão medular: revisão sistemática e metanálise. European Journal of Pain, 2017. Disponível em: < https://pubmed.ncbi.nlm. nih.gov/27341614/ >. Acesso em: 26 de maio de 2020.

CHOI, Y. et al. Pilot study of feasibility and effect of anodal transcutaneous spinal direct current stimulation on chronic neuropathic pain after spinal cord injury. Spinal Cord, $2019 \mathrm{v}$. 57, p. 461-470. Disponível em: <https://pubmed.ncbi.nlm.nih.gov/30700853/>. Acesso em: 30 de Agosto de 2020.

DERRY, S. et al. Pregabalina para dor neuropática em adultos. Cochrane Library, 2019. Disponível em: < https://www.ncbi.nlm.nih.gov/pmc/articles/PMC6353204/>. Acesso em: 27 de abril de 2020.

FRANZ, S. et al. Tratamento da dor em indivíduos com lesão medular: diretrizes da sociedade médica de língua alemã para lesão medular. German Medical Science, 2019. Disponível em: < https://www.ncbi.nlm.nih.gov/pmc/articles/PMC6637293/ >. Acesso em: 27 de abril de 2020 .

GIBSON, W. et al. Transcutaneous electrical nerve stimulation (TENS) for neuropathic pain in adults. The Cochrane Database of Systematic Reviews, 2017. Disponível em: < https:// pubmed.ncbi.nlm.nih.gov/28905362/>. Acesso em: 17 de Novembro de 2020.

GUY, SD. et al. The canpain SCI clinical practice guidelines for rehabilitation management of neuropathic pain after spinal cord: recommendations for treatment. Practice Guideline, 2016 v. 54, p. 14-23. Disponível em: < https://pubmed.ncbi.nlm.nih.gov/27444715/>. Acesso em: 17 de Novembro de 2020.

HAGEN, E. et al. Management of neuropathic pain associated with spinal cord injury. Pain and Therapy, 2015, v. 4, p. 51-65. Disponível em: < https://www.ncbi.nlm.nih.gov/pmc/ articles/PMC4470971/>. Acesso em: 15 de Outubro de 2020.

HARVEY, L. et al. The effectiveness of 22 commonly administered physiotherapy interventions for people with spinal cord injury: a systematic review. Spinal Cord, 2016. Disponível em: < https://www.nature.com/articles/sc201695/>. Acesso em: 16 de Novembro de 2020.

JENDELOVA, P. Estratégias terapêuticas para lesão medular. Revista Internacional de Ciências Moleculares, 2018. Disponível em: < https://pubmed.ncbi.nlm. nih.gov/30332844/ >. Acesso em: 28 de maio de 2020.

JOHNSON, M.; MULVEY, M.; BAGNALL, A. Estimulação elétrica nervosa transcutânea (TENS) para dor fantasma e dor no coto após amputação em adultos. Cochrane library, 
2015. Disponível em: < https://www.ncbi.nlm.nih.gov/pmc/articles/PMC7209768/ >. Acesso em: 06 de maio de 2020.

KENNEDY, P; HASSON, L. A relação entre dor e humor após lesão medular. Journal Medula Medular, 2017. Disponível em: < https://pubmed.ncbi.nlm.nih.gov/26869240/ >. Acesso em: 08 de maio de 2020.

KONG, X; GOZANI, S. Eficácia da estimulação elétrica nervosa transcutânea de alta frequência em local fixo na dor crônica: um estudo observacional em larga escala. Journal of Pain Research, 2018, v. 11, p. 703-714. Disponível em: < https://www.ncbi.nlm.nih.gov/pmc/ articles/PMC5898590/>. Acesso em: 17 de Novembro de 2020.

LI, S. et al. A novel nonpharmacological intervention-breathing-controlled electrical stimulation for neuropathic pain management after spinal cord injury - a preliminar study. Journal of Pain Research, 2016. Disponível em: < https://pubmed.ncbi.nlm.nih.gov/ 27843337/> . Acesso em: 12 de Setembro de 2020.

MARLEEN, C. et al. Effect of burst stimulation evaluated in patients familiar with spinal cord stimulation. Neuromodulation: Technology at the Neural Interface, v. 19, 2016. Disponível em: < https://onlinelibrary.wiley.com/doi/abs/10.1111/ner.12429>. Acesso em: 30 de Outubro de 2020.

MEYER, C. et al. Effects of spinal cord and peripheral nerve stimulation reflected in sensory profiles and endogenous pain modulation. The Clinical Journal of Pain, 2019. Disponível em: < https://pubmed.ncbi.nlm.nih.gov/30260842/>. Acesso em: 25 de Setembro de 2020.

MOKHTARI, T. et al. Transcutaneous electrical nerve stimulation in relieving neuropathic pain: basic mechanisms and clinical applications. Current Pain and Headache Reports, 2020. Disponível em: < https://pubmed.ncbi.nlm.nih.gov/32072323/>. Acesso em: 13 de Novembro de 2020.

ÖZKUL, Ç. Et al. Effects of visual illusion and transcutaneous electrical nerve stimulation on neuropathic pain in patientes with spinal cord injury: A randomized controlled cross-over trial. Journal of Back and Musculoskeletal Rehabilitation, 2015, v. 28, p. 709-719. Disponível em: < https://pubmed.ncbi.nlm.nih.gov/25502348/>. Acesso em: 26 de Agosto de 2020.

PIZETTA, G. et al. Epidemiological analysis of spinal cord injury in the city of joinville. Coluna/Columna, v. 19, n. 1, São Paulo, 2020. Disponível em: < https://www.scielo.br/pdf /coluna/v19n1/2177-014X-coluna-19-01-0048.pdf >. Acesso em: 26 de maio de 2020. 
ROEVER, Leonardo. Understanding systematic review studies. Revista da Sociedade Brasileira de Clínica Médica, 2017, v. 15, p. 127-130. Disponível em: https://docs. bvsalud.org/biblioref/2017/11/875614/152_127-130.pdf. Acesso em: 24 de Novembro de 2020.

SILVA, V.; JESUS, C. Características biopsicossociais de pacientes com dor neuropática após lesão traumática da medula espinhal. Relatos de casos. Revista dor, v. 16, n. 3, São Paulo, 2015. Disponível em: < https://www.scielo.br/pdf/rdor/v16n3/pt_1806-0013-rdor-16-030235.pdf >. Acesso em: 26 de maio de 2020.

SILVA, W. et al. Efficacy of eccentric muscle strengthening in lateral tendinopathy of the elbow. Revista Brasileira de Fisiologia do Exercício, 2017 v. 16, n. 4. Disponível em: https://portalatlanticaeditora.com.br/index.php/revistafisiologia/article/view/1345/3322. Acesso em: 12 de Novembro de 2020.

SCHOLZ, J. et al. A classificação IASP de dor crônica para CID-11: dor neuropática crônica. Revista dor, v. 160, p. 53-59, 2019. Disponível em: < https://pubmed.ncbi.nlm.nih.gov/3058 6071/ >.Acesso em: 26 de maio de 2020.

VITALII, C., OLEG, P. The efficiency of transcutaneous electrical nerve stimulation in association with gabapentin in the treatment of neuropathic pain in patients with spinal cord injury. Romanian Journal of Neurology, 2015. Disponível em: < https://www.researchgate.net /publication/287041336_The_efficiency_of_transcutaneous_electrical_nerve_stimulation_in_ association_with_gabapentin_in_the_treatment_of_neuropathic_pain_in_patients_with_spinal _cord_injury>. Acesso em: 02 de Outubro de 2020.

ZEB, A. et al. Effectivensess of transcutaneous electrical nerve stimulation in management of neuropathic pain in patients with post traumatic incomplete spinal cord injuries. Pakistan Journal of Medical Sciences, 2018. Disponível em: <https://www.ncbi.nlm.nih.gov /pmc/articles/PMC6191807/. Acesso em: 18 de Agosto de 2020 